\title{
The Band Electrode: Ongoing Experience with a Novel Turp Loop to Improve Hemostasis in 265 Patients
}

\author{
Oliver Reich ${ }^{a}$ Peter Faul ${ }^{\mathrm{b}}$ \\ aDepartment of Urology, Ludwig-Maximilians University, Munich, and bepartment of Urology, \\ Klinikum Memmingen, Memmingen, Germany
}

\section{Key Words}

Transurethral resection of the prostate $\cdot$ Benign prostatic hyperplasia $\cdot$ Band electrode $\cdot$ Hemostasis

\begin{abstract}
Introduction: Intraoperative bleeding, one of the major complications of conventional transurethral resection of the prostate (TURP), has led to a search for various alternative methods of tissue ablation in patients with benign prostatic hyperplasia. In 1996, we introduced the newly designed Band Electrode, which combines a high degree of resection efficiency with a better hemostasis. Material and Methods: 265 consecutive patients with prostatism underwent TURP with the Band Electrode. This modified loop electrode does not consist of a thin wire but is rather a flat metal band with a width of $1.2 \mathrm{~mm}$. International prostate symptom score (IPSS), Life Quality Index (L), peak urine flow and postvoid residual urine were evaluated pre- and postoperatively. Additionally, electrical parameters have been recorded with a specially designed high-frequency generator. Results: Median IPSS decreased from 23 preoperatively to 8 and 9 at $12(n=194)$ and 24 months $(n=172)$, respectively $(p<0.001)$. Life Quality Index (L) dropped from 4 to 2 and 2, respectively $(p<0.001)$. Peak urine flow increased from $8.2 \mathrm{ml} / \mathrm{s}$ to
\end{abstract}

\section{KARGER}

Fax +41613061234 E-Mail karger@karger.ch www. karger.com
(C) 2004 S. Karger AG, Basel

0042-1138/04/0721-0040\$21.00/0

Accessible online at:

www. karger.com/uin
18.2 (at postoperative day 3), 17.8 and $17.4 \mathrm{ml} / \mathrm{s}$, respectively ( $p<0.001$ ). Median postvoid residual urine decreased from 77 to 15,22 and $21 \mathrm{ml}$, respectively ( $p<$ 0.001). Resected tissue mass averaged 25 (8-102) g, resection time was $36.5(18-82) \mathrm{min}$. Indwelling catheters were removed 32 (24-72) h postoperatively. None of the patients required blood transfusions or showed signs of a TUR syndrome. Despite a 1.3 times higher power need, the total energy application in vivo was comparable to conventional TURP. Conclusions: This simple exchange of active electrodes leads to a superior hemostasis and thus safety in TURP. Resection speed, tissue ablation and total energy need remain identical.

Copyright $@ 2004$ S. Karger AG, Basel

\section{Introduction}

Most experts still regard transurethral resection of the prostate (TURP) as the surgical 'golden standard' treatment for benign prostatic hyperplasia (BPH). However, a considerably high morbidity rate of $18 \%$ has persisted over the last decades [1]. Intraoperative bleeding is one of the major complications of TURP and has led to a search of various alternative methods of prostatic tissue ablation [2-6], which primarily were sought to provide better 
hemostasis. Of these methods, the different laser procedures [2-4] and the more recently introduced vaporization techniques [5] are used most frequently today.

In 1996, we introduced a newly designed resection loop, the so-called Band Electrode (fig. 1) and reported preliminary results on the first 30 patients [7]. Herein, we report our immediate and midterm results of 265 men treated with this device at our institution. To our knowledge, this study represents the largest series of patients undergoing TURP with the Band Electrode for the treatment of lower urinary tract symptoms on the basis of $\mathrm{BPH}$.

\section{Materials and Methods}

In a single-center study, we prospectively analyzed 265 consecutive patients with moderate to severe prostatism on the basis of $\mathrm{BPH}$ who underwent transurethral resection of the prostate with the Band Electrode (Olympus, Winter \& Ibe GmbH, Hamburg, Germany) from November 1996 to June 1999. The operations were performed by two surgeons. The average age of the patients was 72.3 (54-87) years. Preoperatively, 38 of these men had indwelling catheters because of urinary retention. The median prostate volume measured by transrectal ultrasound (TRUS) was $42(16-115) \mathrm{ml}$ preoperatively. Voiding function was evaluated pre- and postoperatively through International Prostate Symptom Score (IPSS) and Life Quality Index (L). Minimum criteria for TURP with the Band Electrode were urinary retention, a peak flow of less than $12 \mathrm{ml} / \mathrm{s}$ and/or urodynamic signs of obstruction. Diagnostic evaluation consisted of patient history, general physical examination, digital rectal examination, uroflowmetry, in selected cases urethrocystogram (suspicion of urethral stricture) and urodynamic examination (suspicion of neurogenic voiding disorder), ultrasound to determine prostate volume (transrectal) and postvoid residual (suprapubic) and finally laboratory evaluation including prostate-specific antigen.

As described above, the Band Electrode has a width of $1.2 \mathrm{~mm}$, a thickness of $0.3 \mathrm{~mm}$ and a diameter of $7.0 \mathrm{~mm}(\mathrm{CH} 27)$ (fig. 1). This loop can be applied to any standard resectoscope. We used a $27 \mathrm{Fr}$ double-sheath rotating continuous flow resectoscope (Olympus). Power was supplied by the ERBOTOM ICC 350 (ERBE Elektromedizin, Tübingen, Germany), a high-frequency (HF) generator which incorporates automatic voltage control (AutoCut) as well as automatic electric arc control (HighCut). In the HighCut setting the generator delivers a stronger vaporization effect. The system was set to both modes, depending on the intraoperative findings. The average power setting was $200 \mathrm{~W}$. However, the unit delivers the precise power required for cutting, thus avoiding unnecessarily high power levels. This feature is not depending on resection speed and depth. The HF voltage, current and power have been recorded by a computerassisted device, especially designed for this evaluation. The TURP procedure was video-monitored by the Olympus Mini-Chip-Camera OTV S4 (Olympus).

Resection techniques with the Band Electrode differed in no way from those used with conventional wire loop electrodes. After initial resection of the middle lobe, the lateral lobes and the ventral region were resected clockwise from the bladder neck to the verumonta-

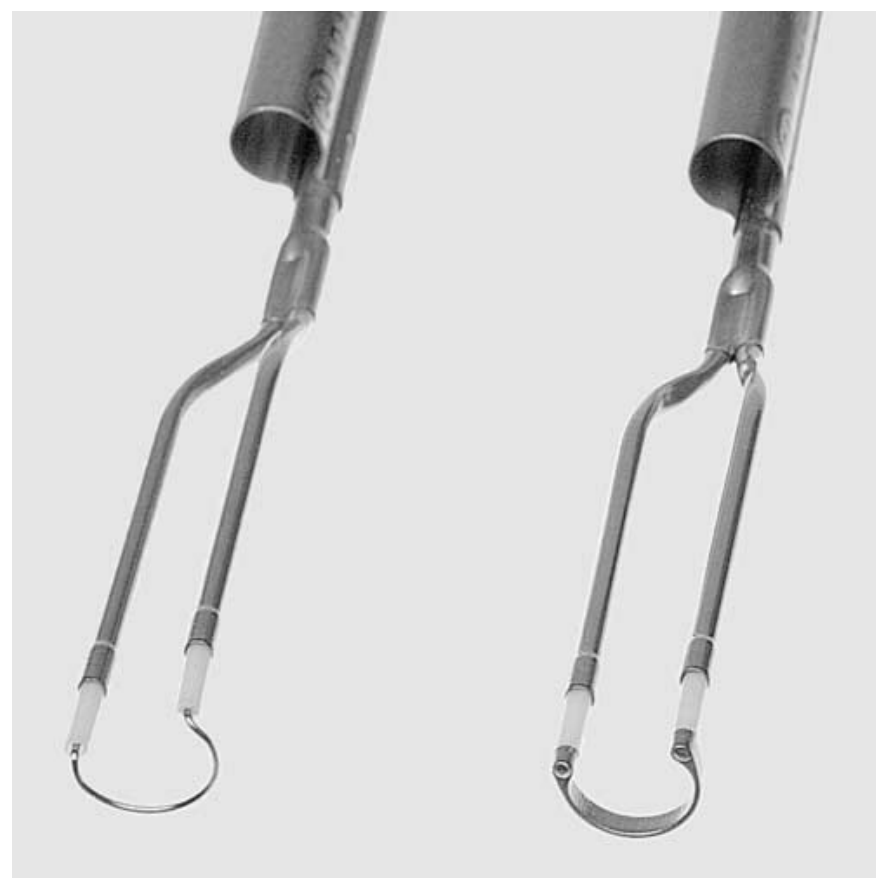

Fig. 1. Comparison of the Band Electrode (right) and a conventional wire loop electrode (left).

num. As a routine, all patients except three with a history of transitional cell carcinoma of the bladder underwent suprapubic cystostomy $(\mathrm{CH} 16)$ preoperatively. Anaesthesia for the TURP procedure was spinal or epidural $(n=179)$ or general $(n=86)$.

Follow-up included IPSS, Life Quality Index (L), uroflowmetry and ultrasonic measurement of postvoid residual urine. Additionally, mass of resected tissue, resection time, catheterization time and hospital days were assessed. Intra- and postoperative blood loss and circulatory complications were evaluated by pre- and postoperative hemoglobin, hematocrit and serum sodium levels.

For statistical analysis, the two-tailed unpaired t test between the different variable parameters was utilized to study unpaired data.

Follow-up was conducted at 3 days $(n=265), 12$ months $(n=194)$ and 24 months $(n=172)$ after surgery. The poor participation of patients at long-term follow-up with only $75 \%$ attendance at 12 months is in part due to the German health care system, which does not routinely cover postsurgical follow-up at our hospital.

\section{Results}

The results in efficacy parameters (table 1) were as follows: median IPSS decreased from 23 (range 15-35) preoperatively to $8(3-16)$ and $9(3-16)$ at 12 and 24 months after surgery, respectively $(\mathrm{p}<0.001)$. Likewise, Life Quality Index (L) dropped from 4 (2-6) to $2(1-3)$ and 2 $(1-4)$, respectively $(\mathrm{p}<0.001)$. As far as urodynamic changes are concerned, peak urine flow improved from 
Fig. 2. Course of power $P$ (left $y$-axis) and energy $E$ (right y-axis) during a typical cut of 2.5 seconds with the Band Electrode (above) and the standard wire loop (bottom). Both cuts were recorded on the same patient at identical cutting speed and depth. Total energy (power.time) was $270 \mathrm{~W}$ (= Joule) for the Band Electrode, compared to $190 \mathrm{~W}$ for the standard loop.
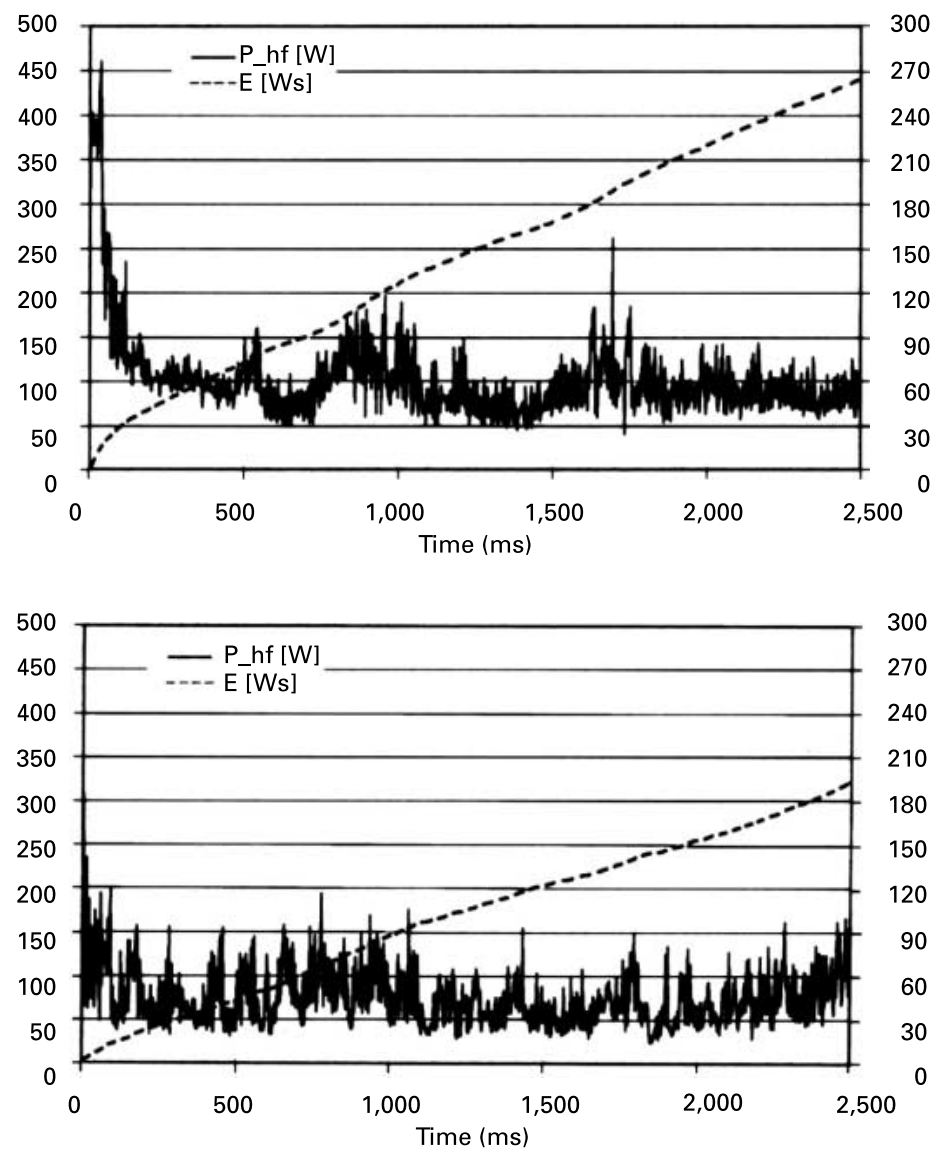

$8.2(0-13)$ to $18.2(11-40) \mathrm{ml} / \mathrm{s}$ at the third postoperative day, and $17.8(9-35)$ and $17.4(8-36) \mathrm{ml} / \mathrm{s}$, respectively $(\mathrm{p}<0.001)$. Median postvoid residual urine decreased from $77(0-345)$ to $15(0-55), 22(0-80)$ and $21(0-95) \mathrm{ml}$, respectively $(\mathrm{p}<0.001)$.

The safety parameters demonstrated the following transformations: Median hemoglobin changed from 15.5 $(13.3-17.0) \mathrm{g} / \mathrm{dl}$ preoperatively to $14.3(10.3-16.0) \mathrm{g} / \mathrm{dl}$ $1 \mathrm{~h}$ after surgery. One day postoperatively, hemoglobin was $14.2(10.8-15.7) \mathrm{g} / \mathrm{dl}$. Hematocrit levels decreased from $44.1 \%(40.6-49.2)$ preoperatively to $40.0 \%$ (36.0$42.6)$ and $40.2 \%$ (36.2-42.9), respectively. Furthermore, serum sodium changed from $143(138-150) \mathrm{mmol} / 1 \mathrm{pre}-$ operatively to $141(127-143) \mathrm{mmol} / \mathrm{l}$ and $141(131-144)$ $\mathrm{mmol} / \mathrm{l}$, respectively.

None of the 265 patients required blood transfusions or showed clinical or laboratory signs of a TUR syn- drome. Only 4 patients had to be recatheterized temporarily due to secondary hemorrhage.

Concerning ablation performance, the resected tissue, weighed instantly after TURP, averaged 25.4 (8-102) g. The mean resection time was 26.4 (7-75) $\mathrm{min}$. This leads to an overall ablation rate of $0.96 \mathrm{~g} / \mathrm{min}$. Indwelling catheters were removed 32 (24-72) h postoperatively. Average stay following TURP was $3.2(1-8)$ days. Regarding the latter, one has to keep in mind that in Germany, most TURP patients will spend more than 7 days in hospital.

Despite a 1.3 times higher power need, the total energy application in vivo was comparable to conventional TURP. For a typical single cut with a duration of more than $1 \mathrm{~s}$, in vivo measurements of electrical parameters revealed a power need averaging $100(85-130) \mathrm{W}$ for the Band Electrode, compared to 75 (60-105) W for a standard TURP wire loop (factor 1.3-1.5). Figure 2 demon- 
Table 1. Changes in efficacy parameters after TURP with the Band Electrode

\begin{tabular}{|c|c|c|c|c|}
\hline & $\begin{array}{l}\text { Preop. } \\
(\mathrm{n}=265)\end{array}$ & $\begin{array}{l}3 \text { days } \\
(\mathrm{n}=265)\end{array}$ & $\begin{array}{l}12 \text { months } \\
(\mathrm{n}=194)\end{array}$ & $\begin{array}{l}24 \text { months } \\
(\mathrm{n}=172)\end{array}$ \\
\hline IPSS & $22.4(15-35)$ & - & $8.3(3-16)^{*}$ & $8.4(3-16)^{*}$ \\
\hline Life Quality Index (L) & $4.2(2-6)$ & - & $1.9(1-3)^{*}$ & $1.8(1-4)^{*}$ \\
\hline Peak urine flow, $\mathrm{ml} / \mathrm{s}$ & $8.2(0-13)$ & $18.2(11-40)^{*}$ & $17.8(9-35)^{*}$ & $17.4(8-36)^{*}$ \\
\hline Postvoid residual urine, $\mathrm{ml}$ & $77(0-345)$ & $15(0-55)^{*}$ & $22(0-80)^{*}$ & $21(0-95)^{*}$ \\
\hline Mass of resected tissue, $\mathrm{g}$ & & $35.3(10-100)$ & & \\
\hline Operation time, $\min$ & & $36.5(18-82)$ & & \\
\hline
\end{tabular}

strates the course of power $(\mathrm{P})$ and energy $(\mathrm{E})$ for both electrodes. To correctly interpret these data, it is essential to remember that the actual power need does not necessarily correspond to the power setting at the generator. On account of less electrode activations with the Band Electrode, the total application of energy was equivalent to standard TURP and averaged approximately $15,000 \mathrm{~W}$ (= Joule) cumulatively, or $500 \mathrm{~W}$ per resected gram of tissue.

Despite the higher power application described above, histological studies were not adversely affected by thermal alterations of resected tissue. In our series, 6 patients were diagnosed for prostate cancer on the basis of TURP specimens.

The use of the wider Band Electrode does not lead to a reduction of the field of vision. The operation site can be judged as accurately and extensively as with the wire loop electrode.

Of the 194 patients available for follow-up at 12 months, $2(1.0 \%)$ developed postoperative urethral strictures, which were successfully treated by urethrotomia interna. None of the patients to date needed reoperation for bleeding or recurrent voiding dysfunction. The complications are summarized in table 2 .

\section{Discussion}

The use of the Band Electrode enables the surgeon to resect hyperplastic prostatic tissue as easily and cleanly as with the conventional wire loop. The same equipment and surgical techniques can be applied. However, the high-frequency generator used in our study permits to carry out the procedure in both, automatic voltage control
Table 2. Complications of TURP with the Band Electrode (12month follow-up)

\begin{tabular}{lll}
\hline & $\begin{array}{l}\text { Number of patients } \\
(196 \text { total })\end{array}$ & Percentage \\
\hline Blood transfusion & 0 & 0 \\
TUR syndrome & 0 & 0 \\
Urethral stricture & 2 & 1.0 \\
Failure to void & 0 & 0 \\
Urinary tract infection & 4 & 2.0 \\
Epididymitis & 1 & 0.5 \\
Urinary incontinence & 0 & 0 \\
\hline
\end{tabular}

mode (AutoCut) as well as automatic electric arc control mode (HighCut). With the AutoCut setting, the resection is performed similarly to conventional TURP, whereas the HighCut setting produces a more vaporization-like resection ('Vapo-TURP'). On account of these features, HighCut was used for the most part of the resection, whereas AutoCut was mainly employed for resection close to the apex. As mentioned above, the width of the metal band does not reduce the surgeon's vision. The resection chips obtained with the Band Electrode are approximately identical in size and shape to those harvested with conventional electrodes, since both loops have a diameter of $7 \mathrm{~mm}$. Histological examination is not altered by thermal changes. This finding was recently confirmed in a study comparing conventional TURP and TURP with a wider electrode, very similar to the Band Electrode [8].

Although our data represent preliminary results with a limited follow-up, the efficacy of the Band Electrode pro- 
Fig. 3. Simplified midline sagittal view of conventional loop (left) and Band Electrode (right) during cutting of a blood vessel within prostatic tissue. Due to the greater width, at identical resection speed, the Band Electrode generates a fourfold longer contact surface between electrode and vessel, resulting in a superior hemostasis.



cedure seems to be equivalent to conventional TURP [1]. This, of course, had to be expected since both techniques are truly tissue-ablative and leave an identical prostatic cavity postoperatively.

The subjective impression of considerably reduced intraoperative bleeding with the use of the Band Electrode is confirmed by the inessential decrease of serum sodium and hematocrit levels. The changes of $2.0 \mathrm{mmol} / \mathrm{l}$ and $4.1 \%$, respectively, are comparable to those reported for $\mathrm{Nd}$ :YAG laser prostatectomies [9] or vaporization procedures [5]. However, it has to be added critically that these parameters depend heavily on infusion therapy. Conventional TURP in large series has reportedly been associated with bleeding requiring blood transfusion in $8.4 \%$, clot retention in $3.3 \%$ and TUR syndrome in $2.1 \%$ [10, 11$]$. None of our 265 patients required transfusions or displayed signs of a TUR syndrome.

Both efficacy and safety parameters generated in our series are well congruent with results of other investigators, using analogous electrodes in smaller studies [12, 13].

The basis for this superior hemostasis is given by the shape of the newly designed electrode. Like conventional wire loop electrodes, the band is approximately $0.3 \mathrm{~mm}$ thick, but its width is nearly $1.2 \mathrm{~mm}$. The duration of electric current at the vessel being coagulated depends on the geometry and size of the electrode and on the speed of cutting. It is proportional to the width of the loop and reciprocal to the speed of cutting. The additional width of the Band Electrode results in a prolonged flow of current, especially into the decisive lateral parts of the prostatic tissue. Since hemostasis is also a function of conduction time, this produces a more effective coagulation of transected bleeding vessels. Every surgeon has experienced the phenomenon of reduced intraoperative bleeding of prostatic tissue when resecting very slowly. Enlarging the width of the electrode from 0.3 to $1.2 \mathrm{~mm}$ creates a fourfold increase in the duration of contact between the electrode and the transected vessels (fig. 3). Thus, in theory the hemostatic effect can therefore be amplified by a factor of four when the speed of resection is constant. Representing even larger surfaces, pure vaporization procedures with roller electrodes perform an even more efficient closure of vessels. However, due to their low ablation efficacy, significantly prolonged activations lead to a 5-10 times higher energy need per removed gram of tissue [14].

Recording electrical parameters during TURP with the Band Electrode and a conventional loop showed that the increase of power and energy applied to the tissue and adherent structures correlates with the width of the electrode. In our studies the power need in the HighCut mode averaged approx. $100 \mathrm{~W}$ for the Band Electrode, compared to approx. $75 \mathrm{~W}$ for the wire loop, representing a 1.3 times higher power application (fig. 2). Concerning each single cut, this factor is also valid for energy application since energy is the product of power and time. Concerning the total energy application per TURP, the 1.3 times higher power need of the Band Electrode was totally compensated by a decreased need for selective coagulation of bleeding vessels.

Nevertheless, the new type of electrode is not capable of closing every vessel at resection speed. Larger vessels still have to be coagulated selectively. Due to their width this is performed more comfortably than with the wire loop. Remarkably, the risk of inadvertent tissue or capsule perforation is also considerably reduced because of the greater width of the new device. 


\section{Conclusions}

In our experience, the Band Electrode represents an ideal synthesis of regular transurethral resection of the prostate (TURP) and the benefits of transurethral vaporization of the prostate (TUVP). Due to its new geometry, it combines conventional resection efficacy with a superior hemostasis. In this initial phase II study displaying outstanding efficacy as well as safety, we showed that the
Band Electrode could play a substantial role in the ongoing effort to raise the golden standard of surgical BPH treatment.

However, definitive evaluation of this promising device will require longer follow-up on a larger patient collective. Therefore, randomized clinical trials in a multicenter setting should be initiated to prospectively compare the Band Electrode with conventional TURP and TUVP.

\section{References}

1 Mebust WK: Transurethral prostatectomy; in Lepor H, Walsh PC (eds): The Urologic Clinics of North America. Philadelphia, Saunders, 1990, pp 575-585.

2 Costello AJ, Bowsher WG, Bolton DM, Braslis $\mathrm{KG}$, Burt $\mathrm{J}$ : Laser ablation of the prostate in patients with benign prostatic hypertrophy. $\mathrm{Br}$ J Urol 1992;69:603-608.

3 Kabalin JN: Laser prostatectomy performed with a right angle firing neodymium:YAG laser fiber at 40 Watts power setting. J Urol 1993; 150:95-99.

4 Muschter R, Whitfield H: Interstitial laser therapy of benign prostatic hyperplasia. Eur Urol 1999;35:147-154.

5 Kaplan SA, Te AE: A comparative study of transurethral resection of the prostate using a modified electro-vaporizing loop and transurethral laser vaporization of the prostate. J Urol 1995; 154:1785-1790.

6 Michel MS, Köhrmann KU, Weber A, Alken P: Rotoresect: New technique for resection of the prostate: Experimental phase. J Endourol 1996;10:473-478.
7 Faul P, Farin G, Reich OM, Steude U: The 'Band Electrode': First experiences with a new TURP procedure to improve hemostasis. Eur Urol 1996;30:403-408.

8 Talic RF, Al Rikabi AC: Transurethral vaporization-resection of the prostate versus standard transurethral prostatectomy: Comparative changes in histopathological features of the resected specimens. Eur Urol 2000;37:301-305.

9 Narayan P, Fournier G, Indudhara R, Leidich $\mathrm{R}$, Shinohara K, Ingerman A: Transurethral evaporation of the prostate (TUEP) with Nd:YAG laser using a contact free beam technique: Results in 61 patients with benign prostatic hyperplasia. Urology 1994;43:813-820.

10 Holtgrewe HL, Mebust WK, Dowd JB, Cockett ATK, Peters PC, Procter C: Transurethral prostatectomy: Practice aspects of the dominant operation in American urology. J Urol 1989; 141:248-253
11 Mebust WK, Holtgrewe H, Cockett A, Peters P, Writing Committee: Transurethral prostatectomy: Immediate and postoperative complications: A cooperative study of 13 participating institutions evaluating 3,885 patients. J Urol 1989;141:243-247.

12 Perlmutter AP, Vallencien G: Thick loop transurethral resection of the prostate. Eur Urol 1999;35:161-165.

13 Kupeli S, Soygur T, Yilmaz E, Budak M: Combined transurethral resection and vaporization of the prostate using newly designed electrode: A promising treatment alternative for benign prostatic hyperplasia. J Endourol 1999;13: 225-228.

14 Reich O, Corvin S, Oberneder R, Sroka R, Muschter R, Hofstetter A: In vitro comparison of transurethral vaporization of the prostate (TUVP), resection of the prostate (TURP), and vaporization-resection of the prostate (TUVRP). Urol Res 2002;30:15-20. 